interaction between famine exposure and genetic risk scores with regard to BMI outcomes. Analyses with weighted risk scores confirm these patterns. Common genetic variants related to BMI do not explain the association between prenatal famine and adult BMI in our study population.

\section{P1-35 ON THE USE OF EMPIRICAL LIKELIHOOD BASED METHODS TO ACHIEVE BALANCE ON MEASURED CONFOUNDERS}

doi:10.1136/jech.2011.142976c.29

G Luta, ${ }^{*}$ A Dragomir, A Barbo, C Loffredo. Georgetown University, Washington, DC, USA

Introduction One of the limitations of the statistical methods that use propensity scores, such as those involving adjustment for the propensity score, matching, subclassification, and inverse probability of treatment weighting, is that they do not achieve exact balance with respect to the measured confounders. Empirical likelihood is a nonparametric method with desirable statistical properties that is perfectly suited to perform the reweighting of the data as to achieve exact balance on measured confounders.

Methods We describe statistical methods that use empirical likelihood to construct weights that add up to one and produce exact balance when applied to the data. For the case involving only categorical confounders, the empirical likelihood based methods produce weights similar to those generated by the inverse probability weighting or standardisation methods. The new methods can handle both categorical and continuous confounders in a unified manner, and allow the incorporation of balancing constraints ranging from simple equalities of means/proportions to more complex constraints related to the comparison of distributions.

Results Under different scenarios of interest, we perform simulations to compare the statistical properties of the proposed method with the inverse probability weighting method. For comparative purposes we also use both methods to evaluate the association between cardiac malformations and birthweight using data from the Washington-Baltimore Infant Study.

Conclusion The proposed empirical likelihood based method performs well and should be used as complementary to the currently available propensity score based methods.

\section{P1-36 DATA SOURCES ON DRUG SAFETY EVALUATION: A REVIEW OF RECENT PUBLISHED META-ANALYSIS}

doi:10.1136/jech.2011.142976c.30

${ }^{1,2} \mathrm{C}$ Alves, ${ }^{1,3} \mathrm{~F}$ Batel-Marques, ${ }^{1,3} \mathrm{~N}$ Craveiro, ${ }^{2,3} \mathrm{~A}$ F Macedo. ${ }^{*}$ School of Pharmacy, University of Coimbra, Coimbra, Portugal; ${ }^{2}$ Health Sciences Research Centre, University of Beira Interior, Covilhã, Portugal; ${ }^{3}$ Central Portugal Regional Pharmacovigilance Centre, AIBILI, Coimbra, Portugal

Introduction Harmful effects of medicines should be reviewed with similar rigour as therapeutic benefits. Most evidence on harms is obtained from post marketing surveillance, so the use of metaanalysis to pool safety information presents challenges of inherent biases and differences in study designs. Yet, it's crucial to provide an accurate safety profile of pharmacological interventions. We aimed to describe the data sources of published meta-analysis of adverse drug effects.

Method We searched meta-analysis published in the last 5 years in six medical journals with the highest impact factor. All the metaanalysis focussing primarily on adverse effects of pharmacological interventions, with pooled results, were included and the characteristics assessed.

Results A total of 61 meta-analysis were included, 16 were published in JAMA, 13 in Lancet, 11 in BMJ, 10 in Ann Intern Med, nine in Arch Intern Med and two in NEJM. Of these $90.2 \%(n=55)$ included only experimental studies, two included only observational studies and four meta-analysis comprised both type of studies. Less than half $(47.5 \% ; n=29)$ of the meta-analysis assessed the quality of the included studies according to specified recommendation statements, and only $18(29.5 \%)$ considered unpublished studies.

Conclusion The majority of meta-analysis of adverse drug effects included only experimental studies, less than half assessed their quality and few considered unpublished studies. These results reinforce the need for methodological research to clarify the role of meta-analysis in Pharmacovigilance and evaluate how to pool safety information from different surveillance methods, to provide an accurate safety profile of pharmacological interventions.

\section{P1-37 WITHDRAWN}

\section{P1-38 MODELLING THE FUTURE BURDENS OF CHRONIC DISEASE THE LESSONS FROM FORESIGHT AND BEYOND}

doi:10.1136/jech.2011.142976c.31

${ }^{1} \mathrm{~T}$ Marsh, ${ }^{*}{ }^{2} \mathrm{~K}$ McPherson, ${ }^{1} \mathrm{M}$ Brown, ${ }^{1} \mathrm{~K}$ Rtveladze. ${ }^{1}$ National Heart Forum, London, UK; ${ }^{2}$ University of Oxford, Oxford, UK

Introduction As the prevalence of chronic diseases continues to climb, the challenges of quantifying the impact of this epidemic to inform decision makers becomes more urgent. Drawing on experiences of work in England, USA, Brazil, Mexico and Russian Federation we will demonstrate how the application of micro simulation modelling can lead to a systematic understanding of the associated morbidities, economic burden and inform policy makers form effective strategies and build the political will for change.

Method The application of micro-simulation modelling techniques to understand the future impact of changes in trends in tobacco consumption and obesity rates and the potential impact of policy interventions.

Results The work initially undertaken for the Foresight Tackling Obesities research was instrumental in galvanising a cross government strategy in England, Healthy Weight, Healthy Lives, subsequent outputs from the simulations in the USA, Brazil, Mexico and Russian Federation should also inform policy in those countries.

Conclusion Morbidity and the economic burden of chronic disease is a practical metric for comparative assessment of health risks, as exemplified by its use by international organizations such as the World Bank, the WHO and the Organisation Economic Co-operation and Development. Nonetheless, the applications for simulation models of morbidity consequences of chronic disease can well go beyond projecting the growth of the problem to the society. A modelling framework provides a useful infrastructure for the comparative evaluation of the effectiveness and return-on-investment of potential policies aimed to alter the drivers and determinants of obesity epidemic.

\section{P1-39 IN RANDOMISATION WE TRUST? RESEARCH REACTIVITY PRODUCES BIAS IN BEHAVIOUR CHANGE TRIALS}

doi:10.1136/jech.2011.142976c.32

\author{
${ }^{1} \mathrm{~J}$ McCambridge, ${ }^{*}{ }^{2} \mathrm{~K}$ Kypri, ${ }^{1} \mathrm{D}$ Elbourne. ${ }^{1}$ LSHTM, London, UK; ${ }^{2}$ University of \\ Newcastle, Newcastle, New South Wales, Australia
}

Introduction Behaviour change trials are increasingly important in public health. Although there has been longstanding awareness of pre-test sensitisation and the Hawthorne effect, the implications of participant reactivity in behaviour change trials are largely unstudied. The aim here is to explore the mechanisms by which biases stemming from the unintended consequences of research participation may be introduced in trials. 